\title{
Biomechanical comparison of locking plate and crossing metallic and absorbable screws fixations for intra-articular calcaneal fractures
}

\author{
Ming $\mathrm{Ni}^{1,2 \dagger}$, Duo Wai-Chi Wong ${ }^{3 \dagger}$, Jiong Mei ${ }^{1}$, Wenxin $\mathrm{Niu}^{1,4^{*}}$ \\ \& Ming Zhang ${ }^{3}$ \\ ${ }^{1}$ Tongji Hospital, Tongji University School of Medicine, Shanghai 200065, China; \\ ${ }^{2}$ Pudong New Area People's Hospital, Shanghai 201299, China; \\ ${ }^{3}$ Interdisciplinary Division of Biomedical Engineering, The Hong Kong Polytechnic University, Hong Kong SAR, China; \\ ${ }^{4}$ Shanghai Yangzhi Rehabilitation Hospital (Shanghai Sunshine Rehabilitation Center), Shanghai 201619, China
}

Received January 14, 2016; accepted March 14, 2016; published online June 27, 2016

\begin{abstract}
The locking plate and percutaneous crossing metallic screws and crossing absorbable screws have been used clinically to treat intra-articular calcaneal fractures, but little is known about the biomechanical differences between them. This study compared the biomechanical stability of calcaneal fractures fixed using a locking plate and crossing screws. Three-dimensional finite-element models of intact and fractured calcanei were developed based on the CT images of a cadaveric sample. Surgeries were simulated on models of Sanders type III calcaneal fractures to produce accurate postoperative models fixed by the three implants. A vertical force was applied to the superior surface of the subtalar joint to simulate the stance phase of a walking gait. This model was validated by an in vitro experiment using the same calcaneal sample. The intact calcaneus showed greater stiffness than the fixation models. Of the three fixations, the locking plate produced the greatest stiffness and the highest von Mises stress peak. The micromotion of the fracture fixated with the locking plate was similar to that of the fracture fixated with the metallic screws but smaller than that fixated with the absorbable screws. Fixation with both plate and crossing screws can be used to treat intra-articular calcaneal fractures. In general, fixation with crossing metallic screws is preferable because it provides sufficient stability with less stress shielding.
\end{abstract}

finite-element analysis, in vitro experiment, calcaneal fracture, plate fixation, absorbable screw, biomechanics

Citation: Ni, M., Wong, D. W. C., Mei, J., Niu, W., and Zhang, M. (2016). Biomechanical comparison of locking plate and crossing metallic and absorbable screws fixations for intra-articular calcaneal fractures. Sci China Life Sci 59, 958-964. doi: 10.1007/s11427-016-0010-9

\section{INTRODUCTION}

Intra-articular calcaneal fractures constitute about $75 \%$ of all calcaneal fractures and are frequently associated with severe functional impairment and disability (Sanders et al., 1993). The goal of surgery is to obtain anatomic reduction while

$\dagger$ Contributed equally to this work

*Corresponding author (email: niu@tongji.edu.cn) retaining the geometry and structure of the calcaneus and restoring the joint axis of the ankle. Selection of the optimal operative technique and modality has been controversial and remains a challenge. Open reduction and internal fixation (ORIF), which includes a plate fixation technique, is the recommended procedure for this fracture (Guerado et al., 2012; Rammelt, 2014). However, some prefer percutaneous reduction and fixation (PRF) with crossing screws, which is indicated for calcaneal fractures with minimal comminution 
(Abdelgaid, 2012; Hammond and Crist, 2013).

Several investigations into the clinical and biomechanical features of both ORIF and PRF revealed the problems associated with the procedures (Folk et al., 1999; Smerek et al., 2008; Tomesen et al., 2011). Metallic implants often fail because of stress shielding (Waris et al., 2004). This has led to the recent proposal of absorbable implants that can be designed with stiffness similar to that of human bone to reduce stress shielding and promote the union of bone (van Dijk et al., 2002). The absorbable materials poly-L-lactic acid (PLLA) and its copolymer have a long history of safe clinical use. PLLA screws have been shown to be sufficiently stable and perform clinically in treating intra-articular calcaneal fractures (Fu et al., 2013; Ni et al., 2015; Zhang et al., 2012).

There have been several studies on the biomechanics of locking/nonlocking plates and comparisons between plate and screw fixations (Redfern et al., 2006; Smerek et al., 2008; Stoffel et al., 2007; Wang et al., 1998), but few reports on the comparison of all these modalities. Finite-element (FE) analysis is a powerful tool for handling clinical biomechanical problems (Huang et al., 2013; Liang et al., 2011; Niu et al., 2014; Ren et al., 2015; Wong et al., 2015). Therefore, the purpose of our study was to evaluate and compare the biomechanics of plate fixation, crossing metallic screw fixation, and crossing absorbable screw fixation by using FE analysis.

\section{RESULTS}

\section{Model validation}

Three-dimensional FE models of calcanei and the fixed fracture models that used (i) locking plate fixation (LPF), (ii) metallic screw fixation (MSF), and (iii) PLLA screw fixation were constructed and validated using in vitro experiments. The FE models and in vitro experiments were based on the same cadaveric sample. FE analysis showed that the intact calcaneus had a higher stiffness value than the fracture models. Of the three fixation modes, the LPF fixation provided the highest stiffness value of $533 \mathrm{~N} \mathrm{~mm}^{-1}$. The vertical stiffness values of the MSF and PLLA fixations were $5 \%$ and $17 \%$, respectively, less than that of the LPF fixation. The vertical construct stiffness of an intact calcaneus and those of the fracture models were compared using the results from cadaveric experiments. As shown in Figure 1, the FE prediction and the experimental measurements had similar trends.

\section{Maximum von Mises stress of fixation implants}

After model validation, the von Mises stress was calculated to assess the failure risk of the implants and bone. Table 1 compares the maximum von Mises stresses on the implants used to fix the calcaneal fracture. The maximum stress on the

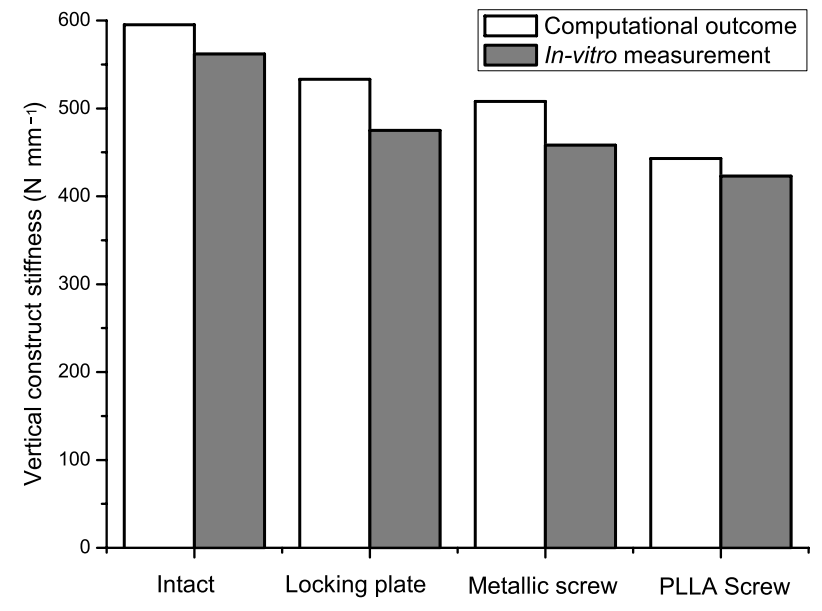

Figure 1 Construct stiffness of intact, locking plate fixation(LPF), metallic screw fixation (MSF), and poly-L-lactic acid (PLLA) screw fixation determined by the computational model and cadaveric experiments.

Table 1 Peak von Mises stress (MPa) on implants and bones in the three fixation models ${ }^{\text {a) }}$

\begin{tabular}{cccc}
\hline Model & Implant & Cortical bone & Cancellous bone \\
\hline LPF & 115.83 & 0.25 & 0.03 \\
MSF & 52.43 & 55.55 & 14.63 \\
PLLA & 21.02 & 55.24 & 16.24 \\
\hline
\end{tabular}

a) LPF, locking plate fixation. MSF, metallic screw fixation. PLLA, polyL-lactic acid.

plate was $115.83 \mathrm{MPa}$, comparatively higher than the stress on the crossing screws. Stress appeared more concentrated on the screw at the sustentaculum tali and where the screws connect the plate. In addition, stress was concentrated at the anterior of the plate, close to the posterior joint facet (Figure 2 ). For the screw fixations, stress was higher around the fracture sites. The peak stress exerted on the metallic screw was much higher than that on the PLLA screw. In both models, the stress on the internal longitudinal screw was higher than that on the lateral screw, and the stress on the inferior transverse screw was higher than that on the superior screw (Figure 2).

The maximum von Mises stresses on the cortical/trabecular calcaneus in the three fixation models are given in Table 1. There was higher stress on both the cortical and trabecular bones with LPF fixation. For the two screw fixations, the stress on the cortical bone was comparable to that with LPF, but the stress on the trabecular bone with PLLA fixation was about $10 \%$ higher than that with MSF.

\section{Fracture micromotion}

The micromotion of fracture in the three fracture models was also analyzed to assess construct stability. The motion with LPF was similar to that with MSF $(0.18 \mathrm{~mm})$. However, in LPF, micromotion was more evident at the sustentacular fragment because it was fixed by a single screw and the fixation 

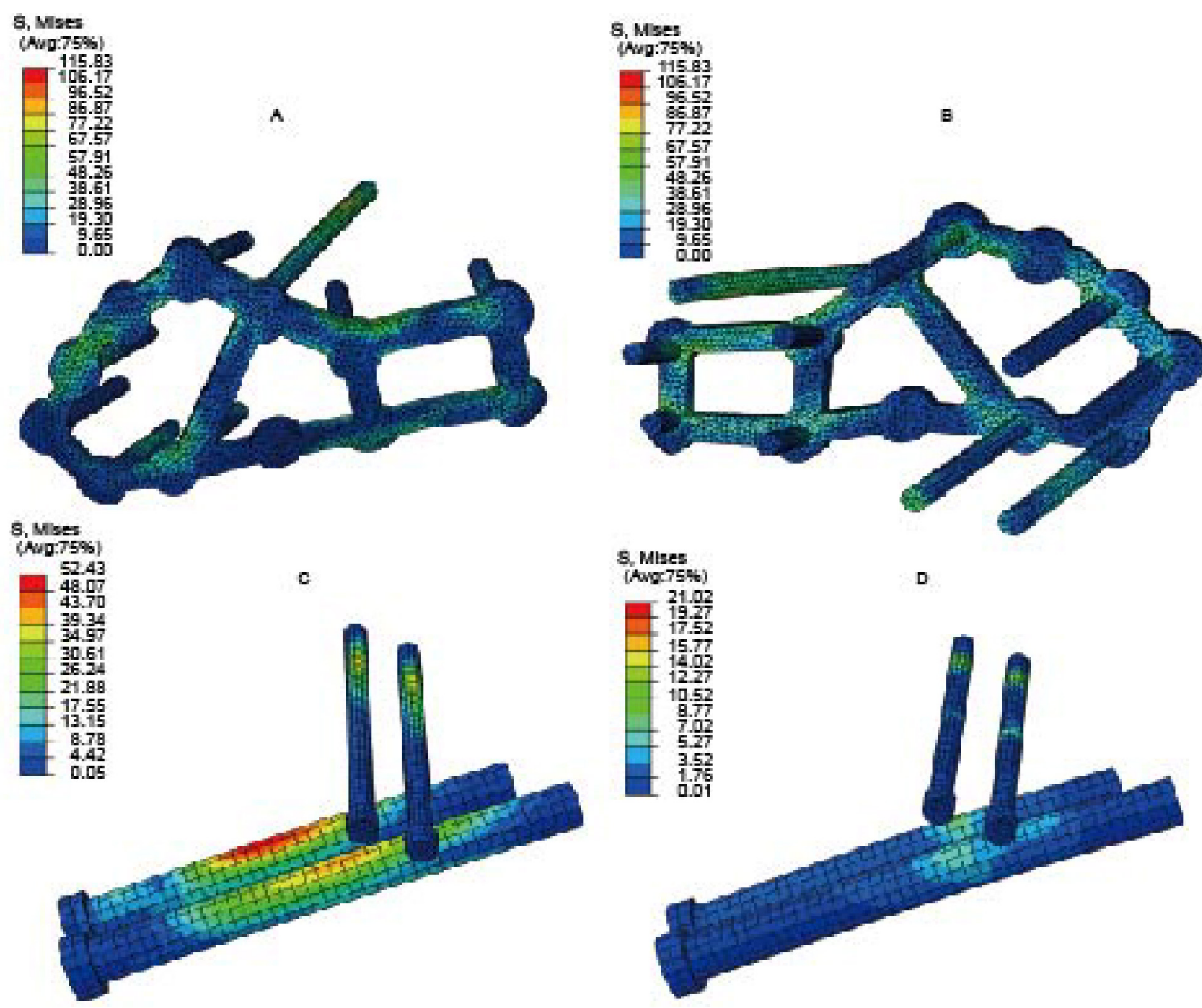

Figure 2 The distribution of von Mises stress (MPa). A, lateral view. B, Medial view in locking plate fixation. C, metallic screw fixation. D, poly-L-lactic acid screw fixation.

was weaker than MSF (Figure 3). The greatest micromotion $(0.30 \mathrm{~mm})$ was with PLLA fixation, most notably close to the anterior and middle fracture sections.

\section{DISCUSSION}

We used a cadaveric experiment to develop and validate a computational model of a Sanders type III fracture, a common type of fracture, the biomechanics of which have been frequently studied (Ni et al., 2015; Smerek et al., 2008; Stoffel et al., 2007; Wang et al., 1998). In this study, we simulated the stance phase of a walking gait, a typical and mechanically demanding loading pattern. Because the foot bears the weight of the entire body during this phase, its simulation would provide reliable information for physicians and patients.

Both FE analysis and the cadaveric experiment demonstrated that the LPF and crossing screws fixations of the calcaneal fractures provided sufficient stiffness. The model was validated prior to analysis of the surgery simulation. The vertical stiffness value predicted by FE analysis was slightly higher than that found by the cadaveric experiment, probably because the cadaveric sample had been embalmed with formalin, which decreased the stiffness of the bone (Öhman et al., 2008).

The locking plate is a commonly used implant for the fixation of calcaneal fractures. In this study, the construct stability of LPF was greater than that of the two crossing screws; it had the smallest displacements at the fracture gaps. Other studies (Nelson et al., 2010; Smerek et al., 2008) found similar results, where LPF of calcaneal fractures provided better fracture reduction than fixation with percutaneous screws. However, this high rigidity promoted a higher concentration of stress on the implant, which, in this situation, could result in hardware failure. Furthermore, higher stress on the locking plate could result in stress shielding of the fractured bones, which would delay healing because of the lack of mechanical stimulation.

Percutaneous screw fixation is an effective treatment for 

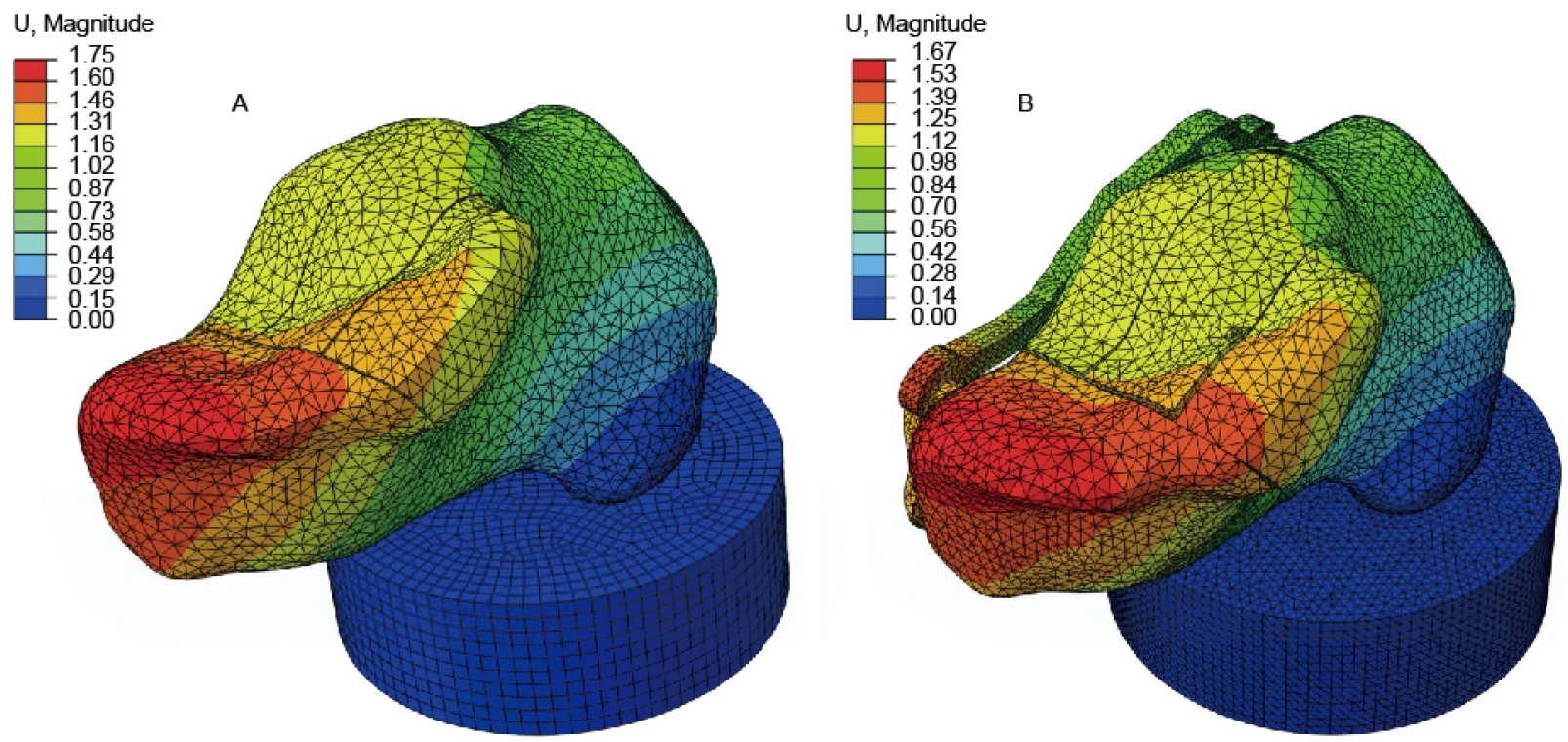

Figure 3 Fracture micromotion (mm) in (A) metallic screw fixation and (B) locking plate fixation.

calcaneal fractures and has minimal skin complications and acceptable clinical outcomes. The technique is suitable for most displaced intra-articular calcaneal fractures and yields favorable clinical results compared to ORIF (Abdelgaid, 2012; Tomesen et al., 2011). Our FE simulation of a crossing screw system showed that it provided satisfactory mechanical strength. The results also showed that the screw material affected the biomechanical performance of the system, e.g., the stress on the metallic screws was much greater and distributed more uniformly than on the PLLA screws.

In PLLA screw fixation, the maximum von Mises stress occurred on the transverse screws, followed by the trabecular bone, with the least amount of von Mises stress on the longitudinal screws. This shows the significance of trabecular bone stiffness in construct stability and that PLLA screws might be less suitable for use in osteoporotic fracture of the calcaneus. Compared with the PLLA screws, the metallic screws can be used in more situations. The high concentration of stress on the medial longitudinal screw and inferior transverse screw suggests that screws with a larger diameter should be used to fix the inferior part of the posterior facet and the medial tuberosity of the calcaneus.

The largest micromotion of the sustentacular fragment occurred with LPF because the fragment was fixed by only one screw and fixation is not strong enough. In addition, the load is distributed asymmetrically on the calcaneus and proportionally on the medial aspect of the posterior facet and the sustentaculum tali (Mahato and Murthy, 2013), causing the sustentacular fragment to rotate along the screw and the fracture to open up. However, the micromotions observed in fixations with screws were much less than that with LPF. Thus, more screws should be used in the lateral plate to fix the sustentacular fragment.
Interfragment micromotion at the fracture site was also crucial to bone healing. Large interfragment movement delays or even prevents the union of the fragments (Claes et al., 1995). In this study, the relative micromovement of fragments in the stance phase of walking was $<0.2 \mathrm{~mm}$ in the LPF and MSF models and $0.3 \mathrm{~mm}$ in the PLLA model. Thus, we can expect bone regeneration when using any of the three fixations.

Because von Mises stress is an equivalent stress, it considers all principal stresses and provides an acceptable yield criterion when the material is assumed isotropic (Doblaré et al., 2004). This criterion was widely adopted for biomechanical analysis (Chen et al., 2014; Liang et al., 2011; Ni et al., 2015; Niu et al., 2014; Tupis et al., 2012). Our simulation showed that the maximum stress exerted on the metallic and absorbable screws was less than the bending strength and shear strength of titanium and PLLA (Chen et al., 2014; Fu et al., 2013; van Dijk et al., 2002). All fixations that we studied were sufficiently stable for treating calcaneal fracture and patients who have these fixations should be encouraged to begin early rehabilitation after surgery. However, no matter which fixation is used, high-impact activities, such as, running or jumping should be avoided because the load on the calcaneus during these activities would be several times that experienced while standing (Giddings et al., 2000).

This study has several limitations. First, only one cadaveric sample was used in the in vitro experiment that validated the FE model. Second, a standard fracture was modeled in the simulation, whereas the direction and nature of real fractures varies depending on the trauma and other factors. Future work should focus on the effect of different fracture patterns on the outcome of operative modalities and protocols.

In conclusion, fixation of a calcaneal fracture by using LPF or crossing screws provides adequate biomechanical stabil- 
ity. The performance of fixation with metallic or absorbable crossing screws was better than that of LPF. MSF predominantly stabilized the fracture gap and reduced the stress on the implant, making it potentially suitable for the treatment of intra-articular calcaneal fractures.

\section{MATERIALS AND METHODS}

\section{Finite-element modeling}

A foot from a Chinese male cadaver (age: 62 years; height: $170 \mathrm{~cm}$; body weight: $70 \mathrm{~kg}$ ) was used in the experiment. All soft tissues were removed, leaving only the calcaneus and talus. The calcaneus underwent a computed tomography (CT) scan by using a slice interval of $0.69 \mathrm{~mm}$ (Brilliance 64 , Philips Electronics, Netherlands). The final CT images had a resolution of $0.54 \mathrm{~mm} \times 0.54 \mathrm{~mm}$. The study protocol was approved in advance by the medical ethical committee of Tongji University.

Based on the CT images, three-dimensional polygon models of the calcaneus were constructed using Mimics 15.0 (Materialise NV, Belgium). A threshold of 600 Hounsfield units differentiated the cortical and trabecular bones (Tupis et al., 2012). The calcaneal models were imported into the FE analysis package ABAQUS 6.14 (Dassault Systèmes, France). Tetrahedral C3D4 elements were used to mesh the model. The calcaneus and the implants were assumed isotropic and linear elastic materials and their material properties, determined in previous reports (Ni et al., 2015; Chen et al., 2014; Moon et al., 2005), are listed in Table 2.

\section{Surgery simulation}

The calcaneus was cut into five fragments to model the Sanders type III fracture described by Smerek et al. Specifically, a V-shaped osteotomy was made near the angle of Gissane, dividing the calcaneus into anterior, middle, and posterior sections. The middle section was further divided into three fragments in the sagittal plane.

Three surgical fixations were performed based on the fracture model: LPF, MSF, and PLLA screw fixation. For LPF, we modeled a lateral anatomical locking plate with screws modeled as 3.5-mm-diameter cylinders. The position and placement of the plate were based on the Arbeitsgemeinschaft für Osteosynthesefragen (AO) technique and clinical experience, with a long screw inserted into the sustentaculum tali

Table 2 Material properties of cortical and cancellous bone and implants (Ni et al., 2015; Chen et al., 2014; Moon et al., 2005) a) $^{\text {a }}$

\begin{tabular}{ccc}
\hline Model part & Young's modulus $(\mathrm{MPa})$ & Poisson's ratio \\
\hline Cortical bone & 10,000 & 0.3 \\
Cancellous bone & 1,450 & 0.2 \\
Metallic material & 110,000 & 0.3 \\
PLLA & 2,500 & 0.2 \\
\hline
\end{tabular}

a) PLLA, poly-L-lactic acid. from the lateral side (Figure 4A). For MSF and PLLA screw fixation, two 7.3-mm-diameter longitudinal screws were inserted from the posteroinferior calcaneal tuberosity into the calcaneal cuboid joint, and two 3.5-mm-diameter transverse screws were inserted from the lateral side beneath the posterior facet into the sustentaculum tali (Figure 4B).

\section{Boundary and loading conditions}

The boundary and loading conditions of the simulation mimicked the stance phase of a walking gait. The posteroinferior calcaneal tuberosity was fixed to a completely restrained base. A vertical load of $700 \mathrm{~N}$ was placed on the superior subtalar articular surfaces of the calcaneus (Figure 5). The coefficient of friction between the fragments and the screws was set at 0.2 (Bulaqi et al., 2015).

\section{Convergence test}

A convergence test was performed by reducing the mesh size until the deviations of the maximum von Mises stress of the implant and the construct stiffness were $<2 \%$. The average mesh size was $1.5 \mathrm{~mm}$. In the crossing screws fixation model, there were 40,301 elements and 127,988 nodes, compared with 67,816 and 145,216 , respectively, in the plate fixation mode.

\section{Model validation}

The cadaveric sample was used in an in vitro experiment to validate the FE model. To simulate the above-mentioned boundary and loading conditions, we used a custom-made clamp. Figure 6 shows that the posteroinferior calcaneal tuberosity was positioned in the clamp by using poly(methyl

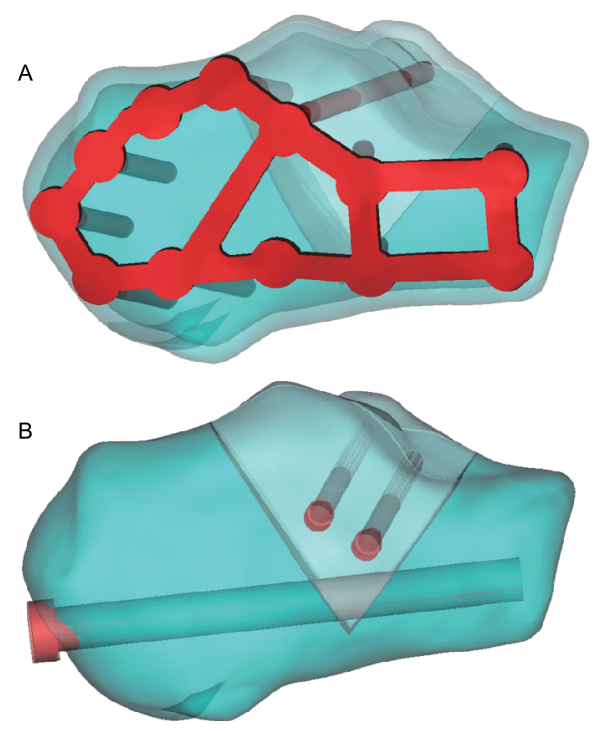

Figure 4 (Color online) The placement and alignment of the fixation implants. (A) locking plate and (B) crossing metallic and crossing absorbable screw fixations. 


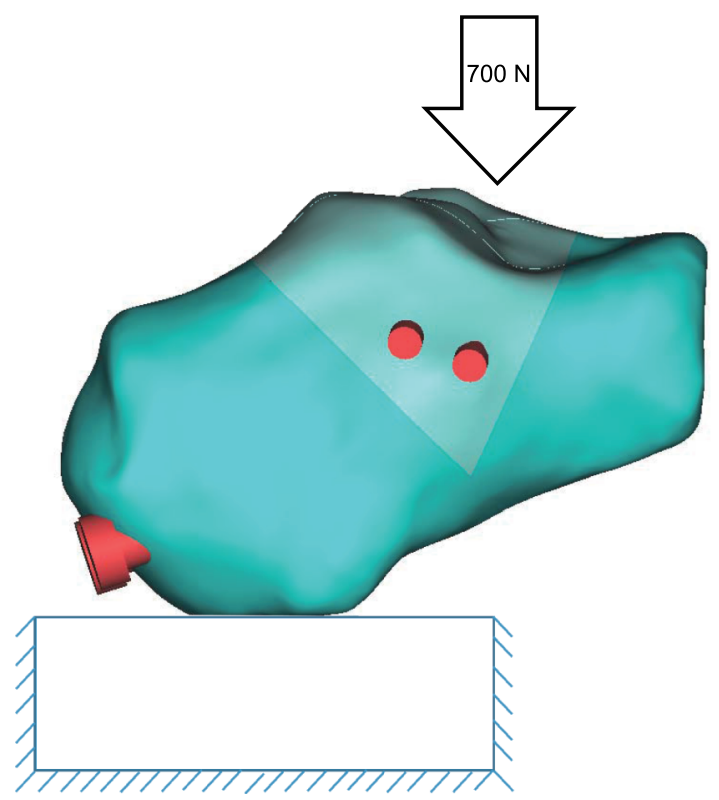

Figure 5 (Color online) Boundary and loading conditions used in the simulation. A 700-N vertical force was applied to the subtalar articular surface, and the posteroinferior calcaneal tuberosity was fixed to a completely restrained base.

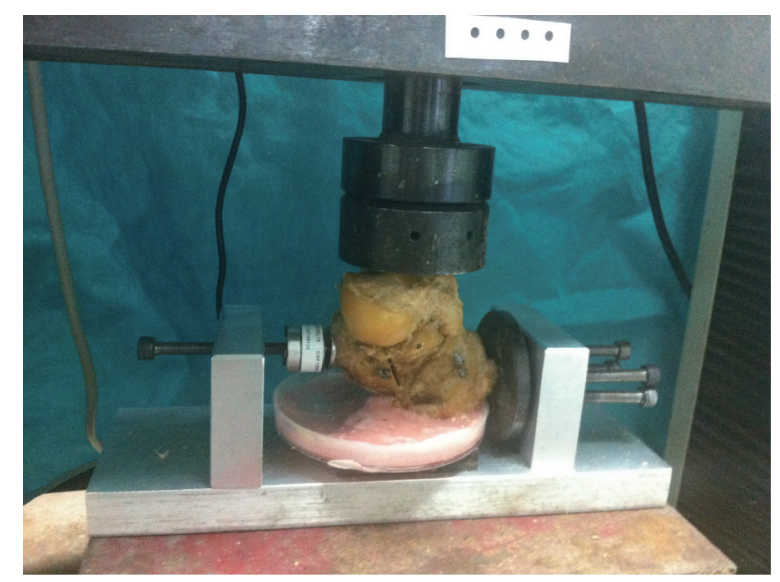

Figure 6 (Color online) In vitro experiment using a material testing machine on a calcaneal specimen.

methacrylate). A mechanical testing machine (CSS-44000; CRIMS Co., Ltd., Changchun, China) applied an axial 700-N load on the talus. The load was placed on the superior surface of the calcaneus according to the anatomical relationship between the calcaneus and the talus. The reproducibility of the loading procedure was tested by loading the same specimen three consecutive times.

Following the experiment, the intact calcaneus was cut according to the protocols used in the surgical simulations detailed above. The fractured specimen underwent three fixation procedures: with a locking plate (Puwei Ltd., Shanghai,
China), four crossing metallic screws (Puwei Ltd.), and four PLLA screws (Dikang Biomedical Co., Chengdu, China). For each surgery, the specimen was subjected to a load as described above. The load displacement of the calcaneus was recorded and the vertical stiffness was calculated for each experiment.

\section{Data analysis}

The construct stiffness, peak von Mises stresses, and fracture micromotions were calculated for each model. The vertical construct stiffness was calculated by dividing the loading force by the maximum vertical displacement of the calcaneus. The fracture micromotion was defined as the relative movement of the initial fracture site after load bearing. It was determined by calculating the mean displacements of the fracture gaps by using 16 pairs of points located along the fracture line at the midpoints and junctions. The average gap enlargement was measured by calculating the displacements of 16 pairs of points.

Compliance and ethics The author(s) declare that they have no conflict of interest.

Acknowledgements This work was supported by the Shanghai Municipal Commission of Health and Family Planning (20144Y0250, 20134Y207), the National Natural Science Foundation of China (11302154, 11272273), and The Hong Kong Research Grant Council GRF (PolyU152216/14E, PolyU5326/11E).

Abdelgaid, S.M. (2012). Closed reduction and percutaneous cannulated screws fixation of displaced intra-articular calcaneus fractures. Foot Ankle Surg 18, 164-179.

Bulaqi, H.A., Mousavi, M.M., Safari, H., Samandari, M.M., and Geramipanah, F. (2015). Dynamic nature of abutment screw retightening: finite element study of the effect of retightening on the settling effect. J Prosthet Dent 113, 412-419.

Chen, S.H., Chiang, M.C., Hung, C.H., Lin, S.C., and Chang, H.W. (2014). Finite element comparison of retrograde intramedullary nailing and locking plate fixation with/without an intramedullary allograft for distal femur fracture following total knee arthroplasty. Knee 21, 224-231.

Claes, L., Wilke, H., Augat, P., Rübenacker, S., and Margevicius, K.J. (1995). Effect of dynamization on gap healing of diaphyseal fractures under external fixation. Clin Biomech (Bristol, Avon) 10, 227-234.

Doblaré, M., Garcia, J., and Gómez, M. (2004). Modelling bone tissue fracture and healing: a review. Eng Frac Mech 71, 1809-1840.

Folk, J.W., Starr, A.J., and Early, J.S. (1999). Early wound complications of operative treatment of calcaneus fractures: analysis of 190 fractures. J Orthop Trauma 13, 369-372.

Fu, X.M., Oshima, H., Araki, Y., Narita, Y., Mutsuga, M., Okada, N., Tsunekawa, T., and Usui, A. (2013). A comparative study of two types of sternal pins used for sternal closure: poly-L-lactide sternal pins versus uncalcined hydroxyapatite poly-L-lactide sternal pins. J Artif Organs $16,458-463$

Giddings, V.L., Beaupré, G.S., Whalen, R.T., and Carter, D.R. (2000). Calcaneal loading during walking and running. Med Sci Sports Exerc 32, $627-634$

Guerado, E., Bertrand, M.L., and Cano, J.R. (2012). Management of calcaneal fractures: what have we learnt over the years? Injury 43, $1640-1650$. 
Hammond, A.W., and Crist, B.D. (2013). Percutaneous treatment of highrisk patients with intra-articular calcaneus fractures: a case series. Injury $44,1483-1485$

Huang, R., Li, X., and Rong, Q. (2013). Control mechanism for the upper airway collapse in patients with obstructive sleep apnea syndrome: a finite element study. Sci China Life Sci 56, 366-372.

Liang, J., Yang, Y.F., Yu, G.R., Niu, W.X., and Wang, Y.B. (2011). Deformation and stress distribution of the human foot after plantar ligaments release: a cadaveric study and finite element analysis. Sci China Life Sci 54, 267-271.

Mahato, N.K., and Murthy, S.S. (2013). Metric analysis of loading magnitudes at articular and non-articular weight-bearing surfaces in human calcaneus. Foot (Edinb) 23, 2-5.

Moon, S.I., Jin, F., Lee, C.J., Tsutsumi, S, and Hyon, S.H. (2005). Novel carbon nanotube/poly (L-lactic acid) nanocomposites; their modulus, thermal stability, and electrical conductivity. Macromol Symp 224, 287-295.

Nelson, J.D., McIff, T.E., Moodie, P.G., Iverson, J.L., and Horton, G.A. (2010). Biomechanical stability of intramedullary technique for fixation of joint depressed calcaneus fracture. Foot Ankle Int 31, 229-235.

Ni, M., Weng, X.H., Mei, J., and Niu, W.X. (2015). Primary stability of absorbable screw fixation for intra-articular calcaneal fractures: a finite element analysis. J Med Biol Eng 35, 236-241.

Niu, W.X., Tang, T.T., Zhang, M., Jiang, C.H., and Fan, Y.B. (2014). An in-vitro and finite element study of load redistribution in the midfoot. Sci China Life Sci 57, 1191-1196.

Öhman, C., Dall'Ara, E., Baleani, M., Van Sint Jan, S., and Viceronti, M. (2008). The effects of embalming using a $4 \%$ formaln solution on the compressive mechanical properties of human cortical bone. Clin Biomech (Bristol, Avon) 23, 1294-1298.

Rammelt, S. (2014). An update on the treatment of calcaneal fractures. J Orthop Trauma 28, 549-550.

Redfern, D.J., Oliveira, M.L., Campbell, J.T., and Belkoff, S.M. (2006). A biomechanical comparison of locking and nonlocking plates for the fixation of calcaneal fractures. Foot Ankle Int 27, 196-201.

Ren, S., Xie, B., Wang, J., and Rong, Q. (2015). Biomechanics of pelvic organ prolapse. Sci China Life Sci 58, 218-220.

Sanders, R., Fortin, P., DiPasquale, T., and Walling, A. (1993). Operative treatment in 120 displaced intraarticular calcaneal fractures results using a prognostic computed tomography scan classification. Clin Orthop Relat Res 290, 87-95.

Smerek, J.P., Kadakia, A., Belkoff, S.M., Knight, T.A., Myerson, M.S., and Jeng, C.L. (2008). Percutaneous screw configuration versus perimeter plating of calcaneus fractures: a cadaver study. Foot Ankle Int 29, 931-935.

Stoffel, K., Booth, G., Rohrl, S.M., and Kuster, M. (2007). A comparison of conventional versus locking plates in intraarticular calcaneus fractures: a biomechanical study in human cadavers. Clin Biomech (Bristol, Avon) 22, 100-105.

Tomesen, T., Biert, J., and Frölke, J. (2011). Treatment of displaced intra-articular calcaneal fractures with closed reduction and percutaneous screw fixation. J Bone Joint Surg Am 93, 920-928.

Tupis, T.M., Altman, G.T., Altman, D.T., Cook, H.A., and Miller, M.C. (2012). Femoral bone strains during antegrade nailing: a comparison of two entry points with identical nails using finite element analysis. Clin Biomech (Bristol, Avon) 27, 354-359.

van Dijk, M., Tunc, D.V., Smit, T.H., Higham, P., Burger, E.H., and Wuisman, P.I. (2002). In vitro and in vivo degradation of bioabsorbable PLLA spinal fusion cages. J Biomed Mater Res 63, 752-759.

Wang, C.L., Chang, G.L., Tseng, C.Y., Yu, W.C., and Lin, R.M. (1998). Strength of internal fixation for calcaneal fractures. Clin Biomech (Bristol, Avon) 13, 230-233.

Waris, E., Konttinen, Y.T., Ashammakhi, N., Suuronen, R., and Santavirta, S. (2004). Bioabsorbable fixation devices in trauma and bone surgery: current clinical standing. Expert Rev Med Devices 1, 229-240.

Wong, D.W., Wang, Y., Zhang, M., and Leung, A.K. (2015). Functional restoration and risk of non-union of the first metatarsocuneiform arthrodesis for hallux valgus: a finite element approach. J Biomech 48, 3142-3148.

Zhang, J., Ebraheim, N., Xiao, B., and Xu, R. (2012). A comparison of absorbable screws and metallic plates in treating calcaneal fractures: a prospective randomized trial. J Trauma Acute Care Surg 72, 106-110.

Open Access This article is distributed under the terms of the Creative Commons Attribution License, which permits any use, distribution, and reproduction in any medium, provided the original author(s) and source are credited. 